\title{
Renormalization group approach to exact sampling
}

\author{
Cédric Chanal and Werner Krauth \\ CNRS-Laboratoire de Physique Statistique, Ecole Normale Supérieure, \\ 24 rue Lhomond, 75231 Paris Cedex 05, France
}

(Dated: November 15, 2018)

\begin{abstract}
In this Letter, we use a general renormalization-group algorithm to implement Propp and Wilson's "coupling from the past" approach to complex physical systems. Our algorithm follows the evolution of the entire configuration space under the Markov chain Monte Carlo dynamics from parts of the configurations (patches) on increasing length scales, and it allows us to generate "exact samples" of the Boltzmann distribution, which are rigorously proven to be uncorrelated with the initial condition. We validate our approach in the two-dimensional Ising spin glass on lattices of size $64 \times 64$.
\end{abstract}

The Markov chain Monte Carlo method [1] has developed into a universal computational approach in many disciplines of science and engineering, and it remains of great importance in the field of statistical physics where it originated more than 50 years ago. Indeed, many difficult calculations in high-dimensional spaces can be expressed (more or less formally) as the calculation of expectation values of an observable $\mathcal{O}$

$$
\langle\mathcal{O}\rangle=\frac{\int \mathrm{d} x \mathrm{e}^{-\beta E(x)} \mathcal{O}(x)}{\int \mathrm{d} x \mathrm{e}^{-\beta E(x)}} \simeq \frac{1}{\tau_{\mathrm{sim}}} \sum_{t=t_{0}}^{t_{0}+\tau_{\mathrm{sim}}} \mathcal{O}\left(x_{t}\right)
$$

To compute the ensemble average on the left of Eq. (1), the Markov chain [on the right of Eq. (11)] passes from one configuration $x_{t}$ at time $t$ to the next one, $x_{t+1}$, most often in a way respecting the detailed balance condition. An ergodic Markov chain, which can eventually reach any configuration $x$ from any other $\tilde{x}$, has the property to visit configurations with probability $\propto \mathrm{e}^{-\beta E(x)}$ in the limit of infinite simulation time $\tau_{\text {sim }} \rightarrow \infty$, where the time average indeed coincides with the ensemble average. In all Monte Carlo calculations, the convergence toward the stationary values of means and (connected) correlation functions is exponential with, for example,

$$
\left\langle\mathcal{O}\left(x_{t}\right) \mathcal{O}\left(x_{t+\tau_{\text {sim }}}\right)\right\rangle_{c} \simeq A \exp \left(-\tau_{\text {sim }} / \tau_{\text {corr }}\right) .
$$

The correlation time $\tau_{\text {corr }}$ provides a crucial scale, because only Markov chain Monte Carlo simulations that have run for times much longer that $\tau_{\text {corr }}$ yield useful results and are essentially free of systematic errors caused by the initial condition.

Data analysis routines allow to reliably estimate the correlation time (and relatedly, the error of a Monte Carlo calculation) whenever $\tau_{\text {corr }} \ll \tau_{\text {sim }}$ (see, for example, 2] for a discussion of the "bunching method"). On the other hand, it is extremely difficult to ascertain that a Monte Carlo simulation has indeed converged, that is, that the essential requirement for its validity, $\tau_{\text {sim }} \gg \tau_{\text {corr }}$ (or at least $\tau_{\text {sim }} \gtrsim \tau_{\text {corr }}$ ), is satisfied. This difficulty is very prominent, for example in the simulation of disordered systems, where the multidimensional space of configurations $x$ is extremely rugged, and where the Monte Carlo dynamics is governed by many different time scales (the longest of which is the correlation time). It is often impossible to assure the validity of a Monte Carlo calculation in disordered systems and other fields without resorting to the comparison with alternative methods, like exact analytic solutions, power expansions, careful finitesize scaling, etc. Bhatt and Young address this point in their classic paper [3] when discussing their convergence test [4]: "In practice, though, one needs a criterion which determines whether any error made in being not quite in equilibrium is acceptably small (...). We know of no "rigorous" such criterion but we have found that the following procedure works well in practice."

More than a decade ago, Propp and Wilson [5] realized a major conceptual breakthrough: they showed how to reformulate the Markov chain Monte Carlo algorithm so that it generates "exact samples" that have no correlation with the initial configuration, not even an exponentially small one, as in Eq. (2). The generation of exact samples is of greatest interest in many real-world applications: it would solve the above-mentioned problem because the samples are exactly in equilibrium (they carry no memory of the initial state) and because the criterion is rigorous.

Unfortunately, Propp and Wilson's procedure, termed "coupling from the past", has been notoriously difficult to apply to complicated physical systems, because it implies, as we will discuss later, the monitoring of the entire configuration space of a physical system under the Monte Carlo dynamics. In the ferromagnetic Ising model above the Curie temperature, the entire configuration space can be monitored, very elegantly, by making use of a partial order of spin configurations $([5]$, see also [2, 6$]$ ). Huber 7] has presented an interesting method that, unlike the partial ordering approach, can be made to work for disordered or frustrated systems [8], but only at very high temperature.

In the present Letter, we show how to apply exact sampling to more complex systems than have been treated before, namely the two-dimensional spin glass on large lattices at low temperature. We use a general, yet rigorous, method which monitors all the configurations in the whole system, not directly (because there are far too many of them) but through "patches" of initially much smaller scale. During the calculation we gradually in- 
crease the size of the patches, not unlike what is done in renormalization-group calculations. At the latest stages of the calculation, the patches are of the same size as the lattice, and the configurations on patches correspond to configurations on the entire system. Our approach to exact sampling works in a wide range of temperatures, and for quite large systems. We are able to judge its efficiency by comparison with a "naive" method which performs a standard Monte Carlo simulation for a representative fraction of all the configurations.

The two-dimensional spin glass does not have a phase transition at temperature $1 / \beta=T>0$, but it is already a quite complex system, due to the presence of disorder. During recent years, the two-dimensional spin glass has been a test bed for new algorithms 9, 10, 11], and has given rise to controversies concerning the specific heat capacity at low temperature [9, 10, 12]. In this model, the convergence time of Monte Carlo calculations is difficult to estimate.

In the heat bath algorithm, the spin $\sigma_{i}(t)$ (on site $i=$ $1, \ldots, N)$ is updated using a uniform random number $\Upsilon_{i}(t)=\operatorname{ran}[0,1]$ :

$$
\sigma_{i}(t+1)= \begin{cases}1 & \text { if } \Upsilon_{i}(t)<\left[1+\mathrm{e}^{-2 \beta h_{i}(t)}\right]^{-1} \\ -1 & \text { else }\end{cases}
$$

where $h_{i}(t)=\sum_{j} J_{i j} \sigma_{j}(t)$ is the local field. The square lattice is bipartite. This allows us to update one entire sublattice simultaneously, in one "sweep", from the spins on the other sublattice, using a vector of random numbers $\Upsilon(t)=\left\{\Upsilon_{1}(t), \ldots, \Upsilon_{N}(t)\right\}$. Two subsequent sweeps update the whole lattice. In the remainder of the Letter, we explain our algorithm for a unique instance of the two-dimensional Ising spin glass, defined by a specific choice of $\left\{J_{i j}= \pm 1\right\}$ for nearest neighbors $i$ and $j$ on the $64 \times 64$ square lattice with periodic boundary conditions. We note that, in principle, any configuration $\boldsymbol{\sigma}$ is described through one sublattice, that is, on $32 \times 64$ sites.

In the "coupling from the past" approach, one considers the simulation as running between an initial configuration, at $t=-\infty$, and the present configuration, at $t=0$ (see Fig. 1). The configuration $\boldsymbol{\sigma}(t=0)$ is an exact sample, because it results from an infinitely long Monte Carlo calculation. It is evidently impossible to perform an infinitely long simulation, but we may pick it up at an intermediate time, $t=t_{0}<0$, where, in principle, the Markov chain could be in any one of the $2^{N}$ configurations, and determine $\boldsymbol{\sigma}(t=0)$, if the Markov chain "couples" between $t=t_{0}$ and $t=0$. This means in our context that under the dynamics of Eq. (3) all the $2^{N}$ possible initial configurations $\boldsymbol{\sigma}\left(t=t_{0}\right)$ yield the same configuration after a finite number $\tau_{\text {coup }}$ of sweeps. If the chain does not couple, we need to complement

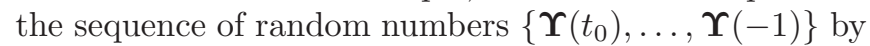
values corresponding to earlier times. The coupling from the past approach to exact sampling relies on the generic property of Markov chains to couple during their evolution [5].

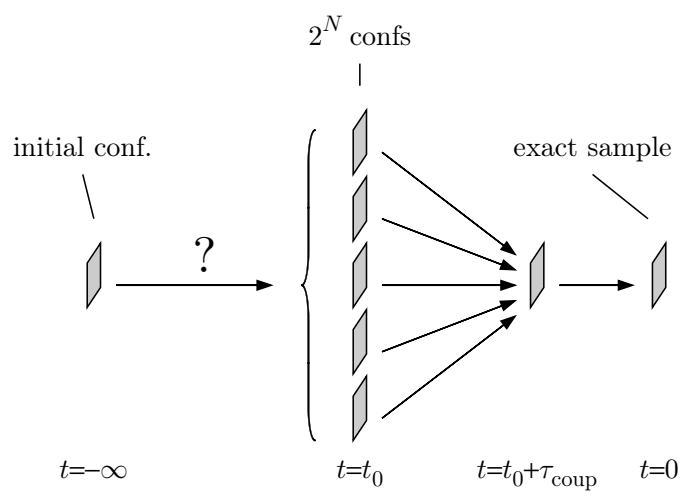

FIG. 1: Schematic representation of coupling from the past: The spin configuration $\boldsymbol{\sigma}(t=0)$ is completely decorrelated from the initial configuration at $t=-\infty$. The configuration $\boldsymbol{\sigma}(0)$ follows from the $2^{N}$ configurations at $t=t_{0}$ if the chain couples between $t=t_{0}$ and $t=0$.

A lower bound on the "coupling time" $\tau_{\text {coup }}$ is obtained by checking that several randomly chosen initial configurations have evolved toward the same state starting from time $t_{0}$. We implement this procedure in a naive

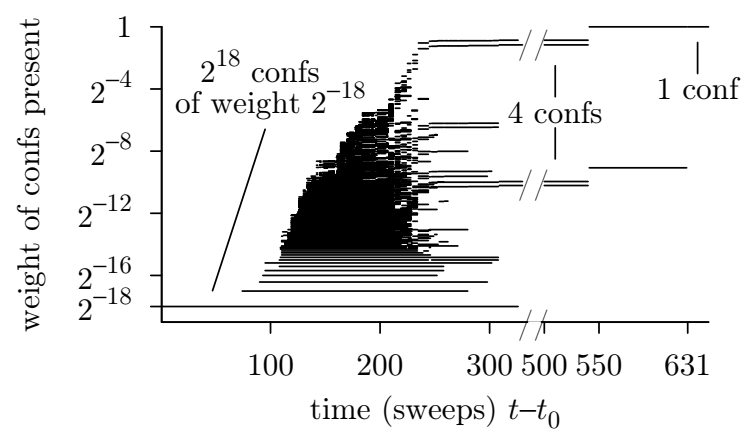

FIG. 2: Weights vs time (in sweeps) of the heat bath algorithm for an instance of the $64 \times 64$ Ising spin glass at $\beta=0.5$ (configurations that couple add weights). At $t-t_{0}=631$, the $2^{18}$ randomly chosen initial configurations have coupled toward a single configuration. Up to time $t \simeq t_{0}+330$, many configurations with very small weights subsist.

algorithm which yield a rigorous value of $\tau_{\text {coup }}$ only if the initial configuration comprise the entire configuration space. We apply the naive algorithm for the present instance of the $64 \times 64$ Ising spin glass with random numbers $\left\{\mathbf{\Upsilon}\left(t_{0}\right), \boldsymbol{\Upsilon}\left(t_{0}+1\right), \ldots\right\}$, for $2^{18}$ initial configurations randomly chosen among the $2^{64 \times 32} \simeq 3.23 \times 10^{616}$ configurations of the entire configuration space. Two different configurations $\boldsymbol{\sigma}(t)$ and $\tilde{\boldsymbol{\sigma}}(t)$ may coalesce at time $t+1$ and will remain the same from then on (see also [13]), so that the number of configurations $\boldsymbol{\sigma}(t)$ decreases with time. We may define the "weight" of a configuration $\boldsymbol{\sigma}(t)$ 
as the fraction of the original configurations at time $t_{0}$ that have evolved towards $\boldsymbol{\sigma}(t)$ (see Fig. 2). In our example, the weight rapidly concentrates on a few configurations, and on a single one after $t-t_{0}=631 \leq \tau_{\text {coup }}$. All weights $\gg 2^{-18}$ in Fig. 2 are expected to remain essentially unchanged for other choices for the $\boldsymbol{\sigma}\left(t_{0}\right)$ and even for an ideal simulation taking into account all the possible initial configurations. At a difference with our renormalization group approach, the naive algorithm cannot yield a rigorous upper bound for $\tau_{\text {coup }}$ and cannot prove that, indeed, $\tau_{\text {coup }}=631$. We note that the coupling time depends on the realization of the Markov chain, that is, all the vectors $\left\{\boldsymbol{\Upsilon}\left(t_{0}\right), \ldots, \boldsymbol{\Upsilon}\left(t_{0}+\tau_{\text {coup }}-1\right)\right\}$.

We now calculate an upper bound for $\tau_{\text {coup }}$ (with given random numbers $\left.\left\{\boldsymbol{\Upsilon}\left(t_{0}\right), \ldots\right\}\right)$. The practical generation of exact samples is straightforward [14, 15].

As shown in Fig. 3, each spin configuration on the entire lattice can be broken up into configurations on patches $k=1, \ldots, N$, that is, pieces of the lattice. We define a set $S_{k}(t)$ of "configurations on patch $k$ " and the product

$$
\begin{array}{r}
\Omega(t)=S_{1}(t) \otimes S_{2}(t) \otimes \cdots \\
\otimes S_{N}(t) /(\text { pairwise compatible }) .
\end{array}
$$

At the initial time $t_{0}$, the set $S_{k}\left(t_{0}\right)$ contains all possible spin configurations on the patch $k$ so that $\Omega\left(t_{0}\right)$ contains a superset of the $2^{N}$ configurations on the entire lattice. At later times $t$, the $S_{k}(t)$ contains a superset of all configurations $\boldsymbol{\sigma}(t)$, restricted to patch $k$. As indicated in Eq. (4), the sets are "pairwise compatible". Pairwise compatibility of $S_{k}(t)$ and $S_{l}(t)$ means that any configuration on patch $k$ must agree in the overlap region $k \cap l$ with at least one configuration (as in Fig. 3) of $S_{l}(t)$ on the neighboring patch $l$. Pairwise compatibility is easy to enforce by a procedure we call "pruning" [16]. It consists in eliminating all spin configurations in $S_{k}(t)$ that lack a compatible configuration in $S_{l}(t)$. However, pairwise compatibility is far from sufficient to assure that all elements in $\Omega(t)$ are valid spin configurations. We may "assemble" two patches $k$ and $l$ by constructing configurations on the patch $k \cup l$ from each pair of compatible configurations of neighboring sets $S_{k}(t)$ and $S_{l}(t)$. In practice, we can assemble all the patches into configurations on the entire lattice only if the sets $S_{i}(t)$ are sufficiently small.

The Monte Carlo algorithm updates a spin on site $i$ as a function of its nearest neighbors. It follows that a spin configuration in $S_{k}(t)$ (the set on patch $k$, of size $m \times m$, at time $t$ ) allows us to determine the spin configuration at time $t+1$ for all the spins on the "center" of the patch, all the sites that do not touch its boundary. In the example of Fig. 3 the patches are of size $4 \times 4$, and their centers of size $2 \times 2$. More generally, the center of an $m \times m$ patch is of size $(m-2) \times(m-2)$.

Many different configurations of $S_{k}(t)$ yield, after one
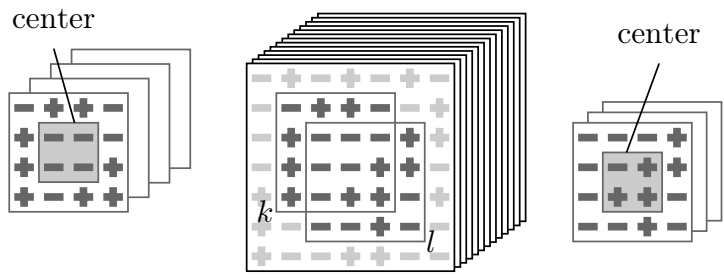

patch $k$

spin configs

patch $l$

FIG. 3: A spin configuration $\sigma=\left\{\sigma_{1}, \ldots, \sigma_{N}\right\}$, and the set of configurations $S_{k}$ and $S_{l}$ on patches $k$ and $l$. The overlap region $k \cap l$ consists here of nine sites. The configuration on the center of a patch, after one sweep, depends on the patch alone.

sweep, identical center configurations. The different configurations on nine neighboring $(m-2) \times(m-2)$ centers can be assembled to form, after pruning, the sets $S_{k}(t+1)$, for $k=1, \ldots, N$. The set $S_{k}(t+1)$ remains a superset of all configurations $\boldsymbol{\sigma}(t+1)$, restricted to patch $k$. Because of the coupling property of the heat bath algorithm, the size of the sets $S_{k}(t+1)$ for small values of $\tau=t-t_{0}$, is generally smaller than the size of $S_{k}(t)$. After many sweeps, the coupling property is offset by the loss of information during the assembly of the centers into the $m \times m$ patches, and the size of the sets $S_{k}(t)$ starts to fluctuate around a constant value. At this point it becomes convenient to assemble patches of size $(m+2) \times(m+2)$, and to apply the above procedure to these larger patches. In this renormalization procedure, the small length scales are effectively "integrated out" by the coupling property of the Monte Carlo algorithm, and one is able to construct the configurations $\boldsymbol{\sigma}(t)$ on ever increasing length scales. The pruning and assembly of patches has been implemented in the PERL programming language using hashing tables and referencing procedures. The programs also transmits pairwise compatibilities from time $t$ to $t+1$. This is highly efficient during the assembly.

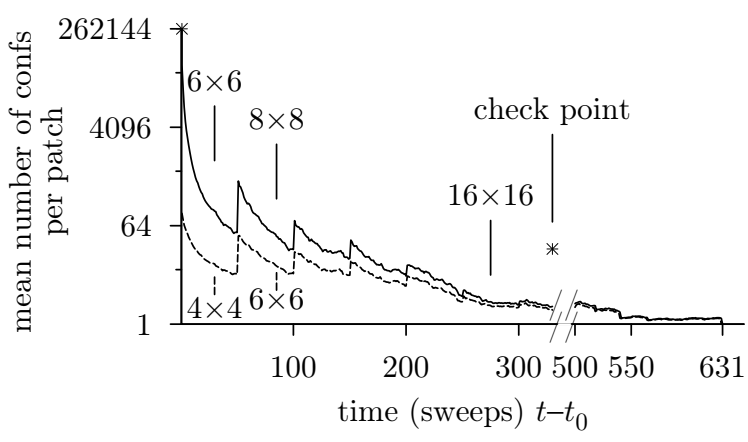

FIG. 4: Coupling of the $2^{64 \times 32}$ configurations of the twodimensional Ising spin glass on a $64 \times 64$ lattice at $\beta=0.5$ followed through patches of increasing size (compare with Fig. 21). 
We now apply the renormalization-group algorithm for determining a rigorous upper bound for $\tau_{\text {coup }}$ to the same instance of the $64 \times 64$ Ising spin glass that was already followed with the naive algorithm in Fig. 2. We follow the evolution of the heat bath algorithm through patches and centers of size $6 \times 6$ and $4 \times 4$, respectively, starting with all the $2^{18}=262144$ possible configurations on each $6 \times 6$ patch at the initial time $t=t_{0}$. We increase the size of the patches after each 50 sweeps (see Fig. 4). At later times, we are able to assemble all configurations on the $64 \times 64$ lattice from the patches. At time $t-t_{0}=330$, for example, the patches are of size $18 \times 18$, and the complete assembly yields 24 configurations $\boldsymbol{\sigma}(t)$ of the $64 \times 64$ system (see the "check point" in Fig. 4). As a consistency check of our algorithm, we verified that these 24 configurations include the 4 configurations present in the naive algorithm at this point (see Fig. 2). The algorithm couples at time $t-t_{0}=631$, that is, it reaches one configuration per patch. During the whole procedure, no configuration was dropped, and therefore, necessarily, the complete assembly of these patches (of size $30 \times 30$ ) yields a single configuration $\boldsymbol{\sigma}\left(t_{0}+631\right)$. In this example, we find that the upper bound for $\tau_{\text {coup }}$ agrees with the lower bound from Fig. 2 .

To conclude our discussion of the instance of the $64 \times 64$ Ising spin glass, we note that our renormalization approach has coupled after the same number of sweeps as the straightforward Monte Carlo calculation of the naive algorithm in Fig. 2. In this sense, the description of spin configurations through the overcomplete set $\Omega(t)$ of Eq. (4I) is efficient, even though all the $2^{32 \times 64} \sim 10^{616}$ configurations that comprise the entire state space of the system have been monitored. However, although both simulations have converged in 631 sweeps of physical time, it should be clear that our renormalization algorithm remains very costly in CPU time (for details see [16]). This was the price to pay in order to exhibit an exact sample.

We have tested our procedure for the Ising spin glass at lower temperatures. At $\beta=0.60$, we checked on many samples of the $J_{i j}$ that we can still couple the Ising spin glass without problems. The number of configurations per $m \times m$ patch levels off after awhile, and the renormalization procedure of Fig. 4 becomes crucial. We have also successfully tested the procedure in the ferromagnetic Ising model below the Curie temperature. In analyzing the behavior of our algorithm, the naive algorithm of Fig. 2 (which generated a sharp lower bound in our spin glass example) becomes a valuable tool.

In conclusion, we have presented a renormalization group approach to exact sampling, and applied it to a large instance of the two-dimensional Ising spin glass. Our approach allows us to control a huge number of con- figurations by means of patches whose sizes increase during the simulation.

Our method can be expected to work generally for models with local interactions but does not rely on special properties, as the partial ordering of configurations, that only hold for severely restricted classes of models. The fact that the model is two-dimensional only simplifies the assembly of local information about the configurations (the patches) but is not required to make it work. We have successfully implemented the approach for the three-dimensional Ising spin glass 16 at temperatures lower than can be handled with a previous method [7, 8].

It would be most exciting if exact sampling could now be applied to even more complex problems, as to the three-dimensional spin glasses around the transition temperature, where the question of whether a Monte Carlo simulation has converged is especially difficult to answer. As mentioned throughout this Letter, the "coupling from the past" approach allows one to draw exact samples (that are proven to have converged) and it puts all Monte Carlo simulations for which it can be applied on an extremely solid foundation. In this Letter we hope to have made a crucial step towards the application of exact sampling to physically challenging problems.

[1] N. Metropolis et al., J. Chem. Phys. 21, 1087 (1953).

[2] W. Krauth, Statistical Mechanics: Algorithms and Computations (Oxford University Press, Oxford, U.K., 2006).

[3] R. N. Bhatt and A. P. Young, Phys. Rev. B 37, 5606 (1988).

[4] R. N. Bhatt, A. P. Young, Phys. Rev. Lett. 54924 (1985).

[5] J. G. Propp and D. B. Wilson, Random Struct. Algorithms 9, 223 (1996).

[6] M. A. Novotny, in Computer Simulation Studies in Condensed Matter Physics XII, edited by D. P. Landau, S. P. Lewis and H. B. Schuettler, Springer Proceedings in Physics Vol. 85 (Springer Verlag, Berlin, 2000) (arXiv:cond-mat/9905195).

[7] M. Huber, Random Struct. Algorithms 22, 43 (2003).

[8] A. M. Childs, R. B. Patterson and J. C. MacKay, Phys. Rev. E 63, 036113 (2001).

[9] L. Saul and M. Kardar, Phys. Rev. E 48, R3221 (1993).

[10] J. S. Wang, R. H. Swendsen, Phys. Rev. B 38, 4840 (1988).

[11] A. Galluccio, M. Loebl, and J. Vondrak, Phys. Rev. Lett. 84, 5924 (2000).

[12] J. Lukic, A. Galluccio, E. Marinari, O. C. Martin and G. Rinaldi, Phys. Rev. Lett. 92, 117202 (2004).

[13] B. Derrida and G. Weisbuch, Europhys. Lett. 4, 657 (1987).

[14] J. A. Fill, Ann. Of Appl. Probab. 8, 131 (1998).

[15] D. B. Wilson, Random Struct. Algorithms, 16, 85 (2000).

[16] C. Chanal and W. Krauth (to be published). 